\title{
CARACTÉRISTIQUES DES PEUPLEMENTS PISCICOLES SENSIBLES AUX ALTÉRATIONS DU MILIEU DANS LES COURS D'EAU DU BASSIN DE LA SEINE.
}

\author{
R. BERREBI dit THOMAS (1), J. BELLIARD (2), P. BOËT (1)
}

(1) Cemagref, Division qualité et fonctionnement hydrologique des systèmes aquatiques, Parc de Tourvoie, BP 44, 92163 Antony Cedex, France.

(2) Conseil Supérieur de la Pêche, Délégation Régionale $n^{\circ} 1,3$ rue Sainte-Marie, 60200 Compiègne, France.

\section{RÉSUMÉ}

Afin de connaître les caractéristiques de la structure des peuplements piscicoles sensibles aux dégradations, nous avons comparé les peuplements de 74 stations faiblement anthropisées à ceux de 133 stations fortement anthropisées. Cette comparaison porte sur 23 paramètres. Ces paramètres caractérisent la structure des peuplements selon leur richesse globale, le régime alimentaire, les habitats d'alimentation, les habitats utilisés au cours du cycle de vie, les substrats de ponte et la capacité de résistance des espèces. Seuls huit paramètres répondent au degré d'anthropisation des cours d'eau. Il s'agit de l'abondance relative des invertivores non résistants, du nombre d'espèces (non résistantes) de fond pierreux, du nombre d'espèces non résistantes de pleine eau, du nombre d'espèces rhéophiles, de l'abondance relative des lithophiles (non résistantes) et du nombre d'espèces sensibles. Ces résultats mettent en évidence la sensibilité des peuplements piscicoles du bassin de la Seine. Ils témoignent également de la difficulté du choix des paramètres d'un indice de qualité basé sur les peuplements piscicoles, en particulier du fait de leur relative pauvreté et de leur faible diversité fonctionnelle dans le bassin de la Seine.

\section{FISH ASSEMBLAGE ATTRIBUTES SENSITIVE TO STREAM ALTERATIONS IN THE SEINE RIVER BASIN (FRANCE).}

\section{ABSTRACT}

To underscore the fish assemblage attributes sensitive to river alterations, we compare 23 parameters of fish assemblages between 74 slightly disturbed sites and 133 strongly disturbed sites. These parameters characterize fish assemblages according to the species richness, the trophic regime, the feeding habitats, the habitat requirements during the life cycle, the spawning substrata and the tolerance capacities of species. Only eight parameters appear to be sensitive to perturbations : the abundance of no tolerant invertivorous individuals, the number of (no tolerant) species feeding on stony substrate, the number of no tolerant species feeding in water column, the rheophilic species richness, the abundance of (no tolerant) lithophilous individuals and the intolerant species richness. These results show that fish assemblages of the Seine River basin are sensitive to river alterations. They also exhibit the difficulty to choose parameters for developing an index based on stream fish communities, 
particularly because of the poor species richness and the low functional diversity of the Seine River basin fish fauna.

\section{INTRODUCTION}

La gestion intégrée des bassins fluviaux nécessite la mise en place d'indicateurs biologiques de la qualité des cours d'eau permettant d'identifier les facteurs responsables des dégradations (KARR, 1993). Dans ce cadre, les peuplements piscicoles constituent des révélateurs synthétiques très significatifs de l'état de l'hydrosystème (FAUSCH et al., 1990). En France, deux méthodes différentes ont été développées pour l'élaboration d'un indice basé sur les peuplements de poissons.

La première a été initiée par les travaux de VERNEAUX (1981) et GRANDMOTTET (1983) et complétée ensuite par DEGIORGI et RAYMOND (1995). Elle permet d'évaluer l'état général des milieux sur la base d'un indice de tolérance/sensibilité des espèces. Toutefois, ces indices ayant été définis de manière globale, sans préciser l'origine de leurs variations (VERNEAUX, 1981), leur utilisation rend difficile tout diagnostique. Ainsi, si la présence d'une perturbation peut être détectée par cette méthode, celle-ci ne permet néanmoins de définir ni sa nature ni son origine.

La deuxième méthode est issue des travaux américains (KARR, 1981) sur les indices de type IBI (Index of Biotic Integrity). Elle a été récemment développée en France par OBERDORFF (OBERDORFF et HUGHES, 1992 ; OBERDORFF et PORCHER, 1994 ; BERREBI dit THOMAS, 1994). Elle est fondée sur l'analyse des variations d'un ensemble de caractéristiques des peuplements piscicoles susceptibles d'être sensibles à différentes modifications du milieu. Cette approche fonctionnelle permet donc d'évaluer d'éventuelles perturbations et de déterminer les compartiments altérés de l'écosystème (KARR et al., 1986 ; MILLER et al., 1988). Néanmoins, cette approche fonctionnelle nécessite la connaissance préalable des réponses des peuplements piscicoles vis-à-vis des différentes perturbations (KARR, 1993).

Actuellement, par manque de connaissances, les caractéristiques des indices sont choisies a priori sur la base du schéma de variation théorique exposé originellement par KARR (HUGHES et OBERDORFF, 1997) pour n'être testées qu'a posteriori lors de l'utilisation de l'indice. Or, cette démarche ne peut être satisfaisante au regard de la différence entre les peuplements nord-américains et ouest-européens, ces derniers étant à la fois moins riches (OBERDORFF et al., 1997) et fonctionnellement moins diversifiés (MAHON, 1984) que les premiers.

Nous nous proposons donc dans cette étude de tester a priori la sensibilité aux dégradations de différentes caractéristiques des peuplements piscicoles du bassin de la Seine. Dans ce but, nous avons comparé l'état de 23 paramètres descriptifs de la structure des peuplements piscicoles de stations au degré d'anthropisation différent.

\section{MATÉRIEL ET MÉTHODES}

\section{Site de l'étude}

Originellement pauvre suite aux dernières glaciations (BANARESCU, 1989), le peuplement du bassin de la Seine ne compte historiquement que 45 espèces (BELLIARD et al., 1995). Parmi ces 45 espèces près de la moitié sont des cyprinidés et 7 , principalement migratrices, ont complètement disparu depuis le siècle dernier du fait de la forte pression anthropique (BELLIARD et al., 1995). 
La Seine a en effet de tout temps subi l'influence de l'homme. D'abord faible au Néolithique, cette influence s'est accentuée à partir du $19^{\mathrm{eme}}$ siècle avec le début de la chenalisation des parties aval (BELLIARD, 1994) pour être actuellement, suite à l'accroissement de la population, l'une des plus fortes du monde : le nombre d'habitants de son bassin rapporté à son débit moyen annuel place la Seine au deuxième rang derrière la Tamise et devant le Nil (LAMONTAGNE et BARIBEAU, 1992). La surface du bassin de la Seine de $73700 \mathrm{~km}^{2}$ concentre en effet 17 millions d'habitants, $40 \%$ des activités industrielles du territoire français, et $50 \%$ du trafic fluvial national. Les principales perturbations proviennent de la gestion des barrages-réservoirs, des opérations de curage et de recalibrage à vocation hydroagricole, des aménagements pour la navigation et pour l'urbanisation, des extractions de granulats et des rejets polluants (AREA, 1992). Cette pression a d'ailleurs atteint un degré tel aujourd'hui qu'il est impossible de trouver des rivières proches d'un état subnaturel dans les parties aval du bassin.

Géographiquement, malgré l'homogénéité des caractéristiques climatiques et morphogéologiques du bassin de la Seine (bassin sédimentaire d'altitude inférieure à $200 \mathrm{~m}$ pour la plus grande partie), le peuplement présente des différences régionales (BELLIARD et al., 1997). Afin de s'en départir, nous avons limité notre étude à l'ensemble le plus homogène du bassin constitué par les deux régions centrales : l'auréole du Crétacé supérieur et la zone centrale à sédiment tertiaire (Figure 1).

Cette situation nous a conduit à sélectionner 207 stations situées sur des rivières drainant des bassins versant de surface inférieure à $4140 \mathrm{~km}^{2}$ (Figure 1).

\section{Description des stations}

Chaque station est définie par sa position dans le gradient amont/aval et son degré d'anthropisation.

Dans la zone médio-européenne, la taille du cours d'eau et la pente sont les deux facteurs prépondérants d'organisation longitudinale des peuplements piscicoles (HUET, 1949). Par conséquent, nous avons choisi ces deux variables pour décrire la position des stations dans le gradient amont/aval. La pente a été calculée à partir de cartes au 1/25000. La taille de la rivière est estimée par la surface de son bassin versant. Le bassin versant de chaque station a été mesuré à l'aide d'un planimètre sur des cartes au 1/500 000 .

Le degré d'anthropisation (faible ou fort) des stations a été défini à partir des données de qualité d'eau (4 classes : excellente, bonne, moyenne et mauvaise) et d'habitat (4 classes : naturel, pseudo-naturel, influencé ou artificialisé) de la cartographie de synthèse des schémas départementaux de vocation piscicole (AREA, 1992). Dans cette cartographie, la qualité de l'eau est estimée à partir de 4 paramètres physico-chimiques (oxygène dissous, DBO5, DCO, ammoniac) et celle de l'habitat à partir de l'état de cinq composantes de l'habitat aquatique (le régime des débits, le profil du lit mineur, les berges et les substrats, l'écoulement et le lit majeur) selon la démarche proposée par TROCHERIE et al. (1985). Sur la base de ces classifications, nous avons considéré comme stations faiblement anthropisées les 74 stations présentant à la fois une qualité d'eau excellente ou bonne et une qualité d'habitat naturel ou pseudo-naturel. Les 133 stations qui présentent une altération importante soit de la qualité d'eau, soit de la qualité de l'habitat, soit des deux compartiments à la fois, sont définies comme fortement anthropisées.

Ces deux catégories de station sont comparables vis-à-vis de leur position au sein du gradient amont/aval : les moyennes des surfaces de bassin versant et des pentes ne sont pas significativement différentes entre les stations faiblement et fortement anthropisées (test-t sur les surfaces de bassin versant, $p=0,39$ et sur les pentes, $p=0,69$ ). 


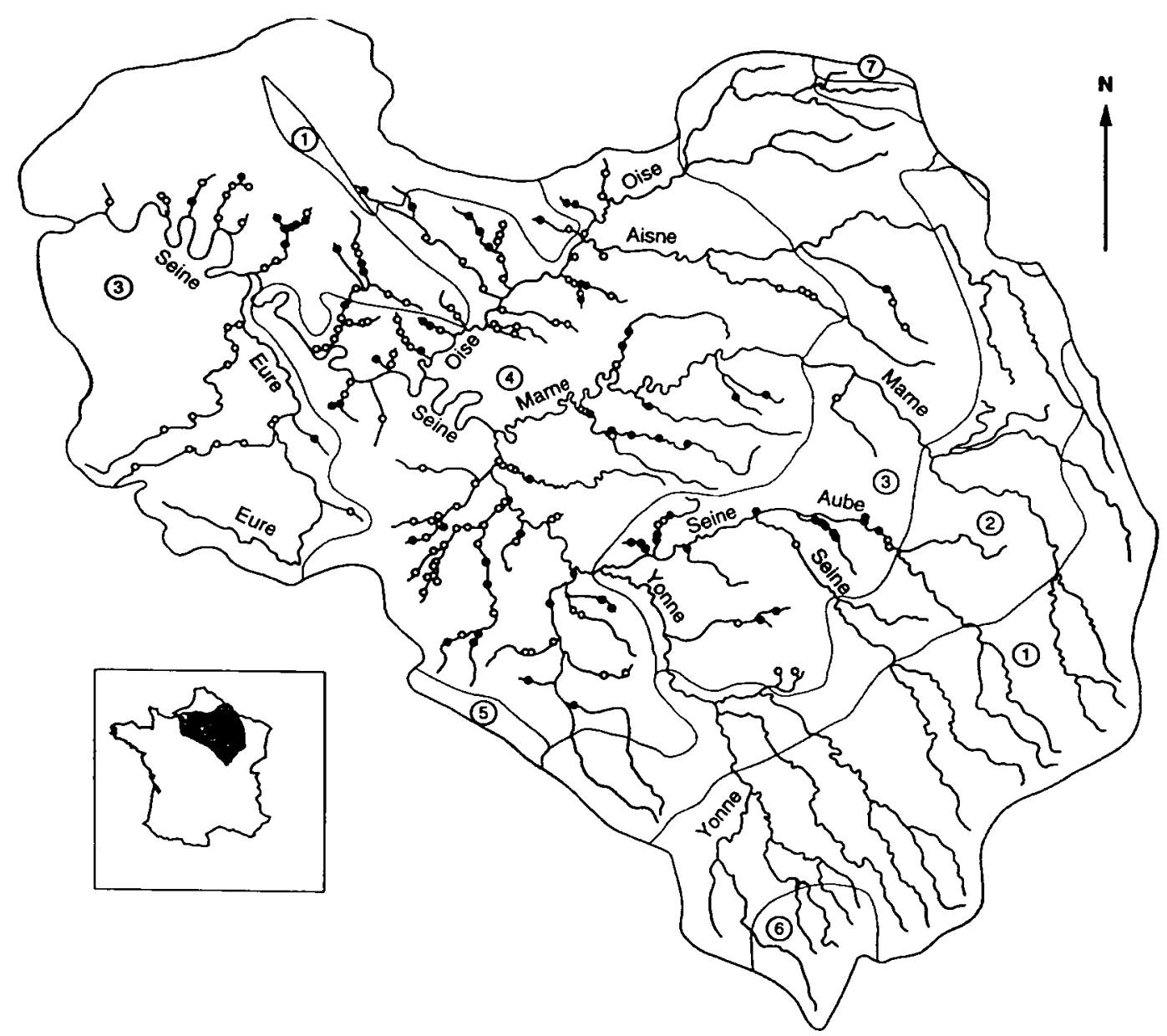

Figure 1

Carte des 207 stations (point plein : station faiblement anthropisée ; point vide : station fortement anthropisée) et des régions naturelles du bassin de la Seine (1: auréoles externes du bassin parisien ; 2 : auréole du Crétacé argileux ou sableux ; 3 : auréoles du Crétacé supérieur ; 4 : zone centrale à sédiment tertiaire ; 5 : zones à couverture détritique ; 6 : socle cristallin et zones volcaniques ; 7 : Ardennes françaises) (d'après DUPIAS et REY, 1985).

\section{Figure 1}

Map of the 207 sites (black point : slightly disturbed site ; white point : strongly disturbed site) and the natural regions of the Seine River basin (1: external aureole of Paris basin ; 2 : aureole of lower Cretaceous ; 3 : aureoles of upper Cretaceous ; 4 : central tertiary sediment zone ; 5 : detritic zones ; 6 : cristallyne socle and volcanic zones ; 7 : French Ardennes) (after DUPIAS and REY, 1985).

\section{Échantillonnage}

Chacune des 207 stations a été échantillonnée une fois par pêche électrique entre 1980 et 1990. Les échantillonnages ont été effectués sur l'ensemble des habitats représentatifs des stations, soit à pied sur tout le lit mineur, soit plus rarement par bateau le long des berges, lorsque la profondeur de la rivière était trop importante.

Cette différence de méthode est susceptible d'introduire des biais dans les captures les échantillonnages des milieux profonds ne peuvent être aussi exhaustifs que ceux des milieux peu profonds. Toutefois, ce biais étant constant, it n'intervient que de manière 
relative dans les comparaisons entre peuplements. De plus, quelle que soit la méthode, tous les types d'habitat représentatifs étant systématiquement prospectés, toutes les espèces présentes sont susceptibles d'être capturées quelle que soit la station.

L'effort d'échantillonnage, que l'on peut estimer par la durée de pêche, est indépendant du degré d'anthropisation des stations. Les durées de pêche des 55 échantillonnages pour lesquels elles sont connues, ne sont en effet pas significativement différentes entre les 2 catégories de station (test-t sur les 55 pêches, 22 stations faiblement anthropisées, 23 fortement anthropisées, $p=0,66$ ).

Finalement, pour minimiser l'effet d'éventuels biais dûs à l'échantillonnage, seules les richesses spécifiques et les abondances relatives d'espèces ou groupes d'espèces (nombre d'individus de l'espèce ou du groupe d'espèces ramené au nombre d'individus total capturés) ont été utilisées.

Ces 207 pêches ont permis la capture de 47102 individus représentant 37 espèces (Figure 2 et Tableau I).

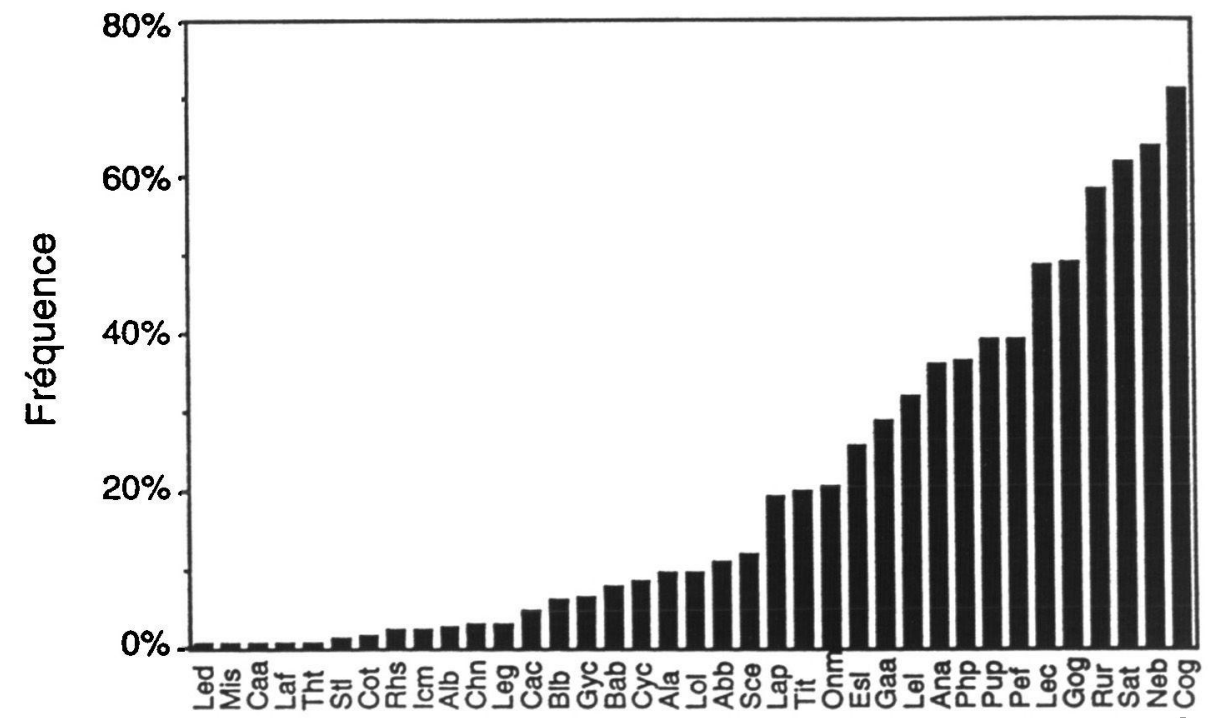

\section{Espèce}

\section{Figure 2}

Fréquence d'occurrence des 37 espèces au sein des 207 échantillonnages (voir Tableau I pour les codes espèces).

\section{Figure 2}

Frequency of the 37 species among the 207 samples (see Table I for the species codes).

\section{Statuts des espèces}

Ce présent travail repose sur une classification préalable des espèces en groupes homogènes (Tableau I) qui concernent : leur origine autochtone/allochtone (BELLIARD, 1994), leur régime trophique (invertivore, piscivore ou omnivore) (MICHEL et OBERDORFF, 1995), leur habitat d'alimentation (espèces prospectant les fonds pierreux ou s'alimentant en pleine eau) (GRANDMOTTET, 1983), les habitats nécessaires à l'accomplissement de leur cycle de vie (rhéophile, limnophile ou eurytope) (SCHIEMER et WAIDBACHER, 1992), leur substrat de ponte (lithophile, phytophile ou phyto-lithophile) 
(BALON. 1975b : BALON, 1981), leur capacité de résistance (sensible ou résistante) (VERNEAUX, 1981). La définition des statuts de certaines espèces a été précisée le cas échéant par des renseignements complémentaires issus de SPILLMANN (1961).

Les espèces ont été considérées comme résistantes ou sensibles selon qu'elles avaient un indice calculé par VERNEAUX (1981) respectivement $\geq$ à 7 ou $\leq$ à 4.5 (Tableau I). Le gardon a été consideré comme lespèce la plus résistante (OBERDORFF et HUGHES. 1992) et la plus généraliste (SCHIEMER et WIESER, 1992) du bassin.

\section{Paramètres étudiés}

23 paramètres ont été étudiés définissant six caractéristiques de la structure des peuplements piscicoles : (i) la richesse globale des peuplements (3 paramètres), (ii) l'équilibre trophique du peuplement (4 paramètres), (iii) l'habitat d'alimentation (4 paramètres), (iv) les habitats utilisés au cours du cycle de vie ( 3 paramètres), (v) les substrats de ponte (5 paramètres) et (vi) la capacité de résistance des espèces (4 paramètres) (Tableau II).

Pour chaque caractéristique, les paramètres sont construits sur des groupes d'espèces au statut équivalent. Toutefois, cinq paramètres fondés sur des espèces à comportement spécialisé, Abln, $\mathrm{NeFp}, \mathrm{NePe}$, AbLit et AbPhy, ont été transformés par élimination des espèces résistantes et appelés respectivement AblnNr, NeFpNr, NePeNr, AbLitNr et AbPhyNr (Tableau II). Cette transformation permet d'éviter que la sensibilité d'un paramètre soit masquée par l'hétérogénéité trop importante des espèces qui le représentent (ANGERMEIER et KARR, 1986 ; STEEDMAN, 1988).

Nous avons également défini les " espèces résistantes euryèces " (AbReu) (Tableau II) comme le groupe d'espèces à la fois résistantes et ne présentant aucune exigence particulière vis-à-vis de leur substrat de ponte, des habitats utilisés au cours de leur vie et de leur régime alimentaire (Abramis brama. Rutilus rutilus et Alburnus alburnus) (Tableau I).

Les paramètres concernant la richesse globale et les capacités de résistance des espèces sont des indicateurs généraux de la dégradation des milieux (KARR, 1981 ; VERNEAUX, 1981).

A l'inverse, les quatre autres caractéristiques ont une signification fonctionnelle. Les paramètres reposant sur des espèces spécialisées dans l'exploitation d'une même ressource (Abln, AblnNr, AbPi, NeFp, NeFpNr, NePe, NePeNr, NeRh, NeLi, AbLit, AbLitNr, AbPhy, AbPhyNr) sont supposés diminuer avec la perte ou la dégradation de cette ressource. Inversement, les paramètres fondés sur des espèces à faible exigence fonctionnelle (AbOm, NeEu, AbPhyLit) sont supposés augmenter avec ces pertes ou dégradations. De nombreuses références bibliographiques font état de tels comportements (Tableau II).

\section{Analyses statistiques}

La sensibilité des paramètres au degré d’anthropisation du milieu est testée en comparant l'ensemble des stations faiblement anthropisées à celui des stations fortement anthropisées. Toutefois, les peuplements piscicoles évoluant le long du gradient amont-aval (HUET, 1949 ;OBERDORFF et al., 1993 ; CHANGEUX, 1995 ; BELLIARD et al.. 1997) nous avons préalablement étudié l'influence de ce gradient sur chacun des paramètres afin de l'éliminer le cas échéant.

Pour chacun des paramètres, l'influence du gradient amont-aval est testée au moyen d'une régression multiple pas à pas utilisant comme variables dépendantes la surface du 


\section{Tableau I}

Liste, code et statut des 37 espèces capturées. Origine (A : allochtone ; N : autochtone), sensibilité ( $\mathbf{S}$ : sensible ; $R$ : résistante), cycle de vie (Rh : rhéophile ; Li : limnophile ; Eu : eurytope), habitat d'alimentation (Fp : fond pierreux ; Pe : pleine eau), substrat de ponte (Litho : lithophile ; Phyto : phytophile ; Phyli : phyto-lithophile) et régime alimentaire ( $\mathrm{I}$ : invertivore ; $\mathrm{O}$ : omnivore ; $\mathrm{P}$ : piscivore).

\section{Table I}

List, code and status of the 37 species caught. Origin (A : allochthonous ; $N$ : autochthonous), tolerance (S : intolerant ; $R$ : tolerant), life cycle (Rh : rheophilic ; Li : limnophilic ; Eu : eurytopic), feeding habitat (Fp : stony substrate ; Pe : water column), spawning substrate (Litho : lithophilous ; Phyto : phytophilous ; Phyli : phytolithophilous) and trophic regime (I : invertivore ; 0 : omnivore ; P : piscivore).

\begin{tabular}{|c|c|c|c|c|c|c|c|}
\hline $\begin{array}{l}\text { Famille } \\
\text { Espece } \\
\end{array}$ & Code & Drigine & Ensibilité & $\begin{array}{l}\text { Cycle } \\
\text { de vie }\end{array}$ & $\begin{array}{c}\text { Habitat } \\
\text { d'alimentation }\end{array}$ & $\begin{array}{c}\text { Substrat de } \\
\text { ponte }\end{array}$ & $\begin{array}{c}\text { Régime } \\
\text { trophique }\end{array}$ \\
\hline $\begin{array}{l}\text { Petromyzontidae } \\
\text { Lampetra fluviatilis } \\
\text { Lampetra planeri }\end{array}$ & $\begin{array}{l}\text { Laf } \\
\text { Lap }\end{array}$ & $\stackrel{N}{N}$ & & Rh & & $\begin{array}{l}\text { Litho } \\
\text { Litho }\end{array}$ & \\
\hline $\begin{array}{l}\text { Anguillidae } \\
\text { Anguilla anguilla }\end{array}$ & Ana & $N$ & $R$ & Eu & & & 1 \\
\hline $\begin{array}{l}\text { Salmonidae } \\
\text { Salmo trutta fario } \\
\text { Oncorhynchus mikiss } \\
\text { Thymallus thymallus }\end{array}$ & $\begin{array}{l}\text { Sat } \\
\text { Onm } \\
\text { Tht }\end{array}$ & $\begin{array}{l}N \\
A \\
N\end{array}$ & $S$ & $\begin{array}{l}\mathrm{Rh} \\
\mathrm{Rh} \\
\mathrm{Rh}\end{array}$ & $\begin{array}{l}\mathrm{Pe} \\
\mathrm{Pe} \\
\mathrm{Pe}\end{array}$ & $\begin{array}{l}\text { Litho } \\
\text { Litho }\end{array}$ & $\begin{array}{l}1 \\
1 \\
1\end{array}$ \\
\hline $\begin{array}{l}\text { Esocidae } \\
\text { Esox lucius }\end{array}$ & Esl & $\mathrm{N}$ & & Eu & $\mathrm{Pe}$ & Phyto & $P$ \\
\hline $\begin{array}{l}\text { Cyprinidae } \\
\text { Leucaspius delineatus } \\
\text { Phoxinus phoxinus } \\
\text { Gobio gobio } \\
\text { Leuciscus leuciscus } \\
\text { Leuciscus cephalus } \\
\text { Chondrostoma nasus } \\
\text { Barbus barbus } \\
\text { Cyprinus carpio } \\
\text { Carassius carassius } \\
\text { Carassius auratus } \\
\text { Tinca tinca } \\
\text { Abramis brama } \\
\text { Blicca bjoerkna } \\
\text { Rutilus rutilus } \\
\text { Scardinius erythrophthalmus } \\
\text { Rhodeus sericeus } \\
\text { Alburnoides bipunctatus } \\
\text { Alburnus alburnus }\end{array}$ & $\begin{array}{c}\text { Led } \\
\text { Php } \\
\text { Gog } \\
\text { Lel } \\
\text { Lec } \\
\text { Chn } \\
\text { Bab } \\
\text { Cyc } \\
\text { Cac } \\
\text { Caa } \\
\text { Tit } \\
\text { Abb } \\
\text { Blb } \\
\text { Rur } \\
\text { Sce } \\
\text { Rhs } \\
\text { Alb } \\
\text { Ala }\end{array}$ & $\begin{array}{l}N \\
N \\
N \\
N \\
N \\
N \\
N \\
N \\
N \\
A \\
N \\
N \\
N \\
N \\
N \\
N \\
N \\
N\end{array}$ & $\begin{array}{l}S \\
S \\
R\end{array}$ & $\begin{array}{l}\mathrm{Li} \\
\mathrm{Rh} \\
\mathrm{Rh} \\
\mathrm{Rh} \\
\mathrm{Rh} \\
\mathrm{Rh} \\
\mathrm{Rh} \\
\mathrm{Eu} \\
\mathrm{Li} \\
\mathrm{Eu} \\
\mathrm{Li} \\
\mathrm{Eu} \\
\mathrm{Eu} \\
\mathrm{Eu} \\
\mathrm{Li} \\
\mathrm{Li} \\
\mathrm{Rh} \\
\mathrm{Eu}\end{array}$ & $\begin{array}{l}\mathrm{Pe} \\
\mathrm{Pe} \\
\mathrm{Fp} \\
\mathrm{Pe} \\
\mathrm{Pe} \\
\mathrm{Fp} \\
\mathrm{Fp}\end{array}$ & $\begin{array}{l}\text { Phyto } \\
\text { Litho } \\
\text { Litho } \\
\text { Phyli } \\
\text { Litho } \\
\text { Litho } \\
\text { Litho } \\
\text { Phyto } \\
\text { Phyto } \\
\text { Phyli } \\
\text { Phyto } \\
\text { Phyli } \\
\text { Phyto } \\
\text { Phyli } \\
\text { Phyto } \\
\text { Litho } \\
\text { Phyli }\end{array}$ & $\begin{array}{l}0 \\
0 \\
0 \\
0 \\
0 \\
0 \\
0 \\
0 \\
0 \\
0 \\
0 \\
0 \\
0 \\
0 \\
0 \\
\\
0 \\
0\end{array}$ \\
\hline $\begin{array}{l}\text { Cobitidae } \\
\text { Cobitis taenia } \\
\text { Nemachelius barbatulus }\end{array}$ & $\begin{array}{l}\text { Cot } \\
\text { Neb }\end{array}$ & $\begin{array}{l}N \\
N\end{array}$ & R & $\begin{array}{l}\text { Rh } \\
\text { Rh }\end{array}$ & $\mathrm{Fp}$ & $\begin{array}{l}\text { Phyto } \\
\text { Litho }\end{array}$ & $\begin{array}{l}1 \\
1\end{array}$ \\
\hline $\begin{array}{l}\text { Ictaluridae } \\
\text { Ictalurus melas }\end{array}$ & $\mathrm{Icm}$ & A & & Eu & & Litho & 0 \\
\hline $\begin{array}{l}\text { Gadiae } \\
\text { Lota lota }\end{array}$ & Lol & $N$ & $\mathrm{~S}$ & $\mathrm{Rh}$ & $\mathrm{Pe}$ & Litho & $P$ \\
\hline $\begin{array}{l}\text { Gasterosteidae } \\
\text { Gasterosteus aculeatus } \\
\text { Pungitius pungitius }\end{array}$ & $\begin{array}{l}\text { Gaa } \\
\text { Pup }\end{array}$ & $\begin{array}{l}N \\
N\end{array}$ & & $\begin{array}{l}\mathrm{Li} \\
\mathrm{Li}\end{array}$ & & & $\begin{array}{l}0 \\
0\end{array}$ \\
\hline $\begin{array}{l}\text { Centrarchidae } \\
\text { Lepomis gibbosus } \\
\text { Micropterus salmoides }\end{array}$ & $\begin{array}{l}\text { Leg } \\
\text { Mis }\end{array}$ & $\begin{array}{l}A \\
A\end{array}$ & $S$ & $\begin{array}{l}\text { Eu } \\
\text { Eu }\end{array}$ & $\begin{array}{l}\mathrm{Pe} \\
\mathrm{Pe}\end{array}$ & $\begin{array}{l}\text { Litho } \\
\text { Litho }\end{array}$ & $\begin{array}{l}1 \\
p\end{array}$ \\
\hline $\begin{array}{l}\text { Percidae } \\
\text { Perca fluviatilis } \\
\text { Stizostedion lucioperca } \\
\text { Gymnocephalus cernua }\end{array}$ & $\begin{array}{l}\text { Pef } \\
\text { Stl } \\
\text { Gyc }\end{array}$ & $\begin{array}{l}N \\
N \\
N\end{array}$ & $\begin{array}{l}\mathrm{R} \\
\mathrm{R}\end{array}$ & $\begin{array}{l}\mathrm{Eu} \\
\mathrm{Eu} \\
\mathrm{Eu}\end{array}$ & $\begin{array}{l}\mathrm{Pe} \\
\mathrm{Pe} \\
\mathrm{Fp}\end{array}$ & $\begin{array}{l}\text { Phyli } \\
\text { Phyli } \\
\text { Phyli }\end{array}$ & $\begin{array}{l}P \\
P \\
O\end{array}$ \\
\hline $\begin{array}{l}\text { Cottidae } \\
\text { Cottus gobio }\end{array}$ & $\operatorname{cog}$ & $\mathrm{N}$ & $\mathrm{s}$ & $\mathrm{Rh}$ & $\mathrm{Fp}$ & Litho & 1 \\
\hline
\end{tabular}


Tableau II

Liste des caractéristiques et des paramètres étudiés. Code des paramètres, sens de variation attendu avec l'augmentation du degré d'anthropisation des milieux et références bibliographiques.

Table II

List of studied characteristics and parameters. Code, expected variation according to the degree of alteration and references.

\begin{tabular}{|c|c|c|c|}
\hline $\begin{array}{l}\text { CARACTÉRISTIQUE } \\
\text { Paramètre }\end{array}$ & Code & $\begin{array}{l}\text { Variation avec } \\
\text { le degré de } \\
\text { dégradation }\end{array}$ & Référence bibliographique \\
\hline $\begin{array}{l}\text { RICHESSE GLOBALE } \\
\text { Nombre d'espèces }\end{array}$ & Nesp & $x$ & $\begin{array}{l}\text { KARR et al. } 1986 \text {; LEONARD et ORTH, } 1986 \text {; } \\
\text { PENAZ ef JURAJDA, } 1993\end{array}$ \\
\hline $\begin{array}{l}\text { Nombre d'espèces } \\
\text { autochtones }\end{array}$ & $\mathrm{NeN}$ & $x$ & $\begin{array}{l}\text { OBERDORFF et PORCHER, } 1994 \text {; } \\
\text { HUGHES et GAMMON, } 1987\end{array}$ \\
\hline Nombre d'espèces allochtones & $\mathrm{NeA}$ & $\lambda$ & $\begin{array}{l}\text { HUGHES et GAMMON, } 1987 ; \text { MILLER et al., } 1988 \text {; } \\
\text { CRUMBY et al., } 1990 ; \text { MINNS et al., 1994; } \\
\text { LEIDY et FIELDER, } 1985\end{array}$ \\
\hline \multicolumn{4}{|l|}{ RÉGIME TROPHIQUE } \\
\hline $\begin{array}{l}\text { Abondance relative des } \\
\text { invertivores }\end{array}$ & Abln & 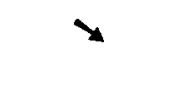 & $\begin{array}{l}\text { KARR et al, } 1986 \text {; OBERDORFF et HUGHES, } 1992 \text {; } \\
\text { GATZ et HARIG, 1993; SCHLOSSER, 1982a; } \\
\text { OBERDORFF et PORCHER, } 1994\end{array}$ \\
\hline $\begin{array}{l}\text { Abondance relative des } \\
\text { invertivores non résistants }\end{array}$ & $\mathrm{Ab} / \mathrm{nNr}$ & $\mathbf{x}$ & \\
\hline $\begin{array}{l}\text { Abondance relative des } \\
\text { piscivores }\end{array}$ & $\mathrm{AbPi}$ & 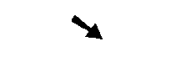 & $\begin{array}{l}\text { KARR et al., } 1986 \text {; OBERDORFF et HUGHES, } 1992 \text {; } \\
\text { SCHLOSSER, } 1982\end{array}$ \\
\hline $\begin{array}{l}\text { Abondance relative des } \\
\text { omnivores }\end{array}$ & AbOm & $\pi$ & $\begin{array}{l}\text { KARR et al., } 1986 \text {; OBERDORFF et HUGHES, } 1992 \text {; } \\
\text { SCHLOSSER, } 1982\end{array}$ \\
\hline $\begin{array}{l}\text { HABITAT D'ALIMENTATION } \\
\text { Nombre d'espèces de fond } \\
\text { pierreux }\end{array}$ & NeFp & & $\begin{array}{l}\text { KARR et al., } 1986 ; \text { OBERDORFF et PORCHER, } 1994 \text {; } \\
\text { LEONARD et ORTH, } 1986\end{array}$ \\
\hline $\begin{array}{l}\text { Nombre d'espèces non } \\
\text { résistantes de fond pierreux }\end{array}$ & NeFpNr & $x$ & \\
\hline $\begin{array}{l}\text { Nombre d'espèce de pleine } \\
\text { eau }\end{array}$ & $\mathrm{NePe}$ & 4 & KARR et al., 1986 \\
\hline $\begin{array}{l}\text { Nombre d"espèces non } \\
\text { résistantes de pleine eau }\end{array}$ & $\mathrm{NePeNr}$ & & \\
\hline $\begin{array}{l}\text { CYCLE DE VIE } \\
\text { Nombre d'espèces rhéophiles }\end{array}$ & $\mathrm{NeRh}$ & 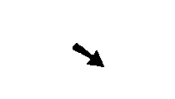 & $\begin{array}{l}\text { SCHIEMER et WAIDBACHER, } 1992 \text {; SCHIEMER et al., } \\
1991 ; \text { PENAZ et JURANDA, } 1993\end{array}$ \\
\hline Nombre d'espèces limnophiles & $\mathrm{NeLi}$ & . & $\begin{array}{l}\text { PENAZ et JURANDA, 1993; SCHIEMER et } \\
\text { WAIDBACHER, } 1992\end{array}$ \\
\hline Nombre d'espèces eurytopes & NeEu & $\pi$ & $\begin{array}{l}\text { SCHIEMER et WAIDBACHER, } 1992 \text {; } \\
\text { PENAZ et JURAJDA, } 1993\end{array}$ \\
\hline $\begin{array}{l}\text { SUBSTRAT DE PONTE } \\
\text { Abondance relative des } \\
\text { lithophiles }\end{array}$ & AbLit & $x$ & $\begin{array}{l}\text { BERKMAN et RABEN, } 1987 \text {; HOLCIK, } 1990 ; \\
\text { OBERDORFF et HUGHES, } 1992 ; \\
\text { GATZ et HARIG, } 1993 \text {; PENCZAK et MANN, } 1993 \text {; } \\
\text { OBERDORFF et PORCHER, } 1994\end{array}$ \\
\hline $\begin{array}{l}\text { Abondance relative des } \\
\text { lithophiles non résistants }\end{array}$ & AbLitNr & $\mathbf{x}$ & \\
\hline $\begin{array}{l}\text { Abondance relative des } \\
\text { phytophiles }\end{array}$ & AbPhy & $x$ & $\begin{array}{l}\text { PENAZ et JURAJDA, } 1993 ; \text { BELLIARD, } 1994 \text {; } \\
\text { JURAJDA, } 1995 ; \text { COPP, } 1991\end{array}$ \\
\hline $\begin{array}{l}\text { Abondance relative des } \\
\text { phytophiles non resistants }\end{array}$ & AbPhyNr & $\mathbf{x}$ & \\
\hline $\begin{array}{l}\text { Abondance relative des phyto- } \\
\text { lithophiles }\end{array}$ & AbPhyLit & $\pi$ & $\begin{array}{l}\text { PENAZ et JURAJDA, } 1993 \text {; BELLIARD, } 1994 \\
\text { PENAZ et al., } 1991\end{array}$ \\
\hline $\begin{array}{l}\text { RÉSISTANCE/SENSIBILITÉ } \\
\text { Nombre d'espèces sensibles }\end{array}$ & $\mathrm{NeS}$ & & $\begin{array}{l}\text { KARR et al., } 1986 ; \text { VERNEAUX, 1981; } \\
\text { LEONARD et ORTH, } 1986\end{array}$ \\
\hline $\begin{array}{l}\text { Abondance relative des } \\
\text { individus résistants }\end{array}$ & $A b R$ & $\pi$ & $\begin{array}{l}\text { OBERDORFF et PORCHER, } 1994 \text {; } \\
\text { GATZ el HARIG, } 1993\end{array}$ \\
\hline $\begin{array}{l}\text { Abondance relative des } \\
\text { individus résistants euryèces }\end{array}$ & AbReu & $\pi$ & KARR, 1981 \\
\hline $\begin{array}{l}\text { Abondance relative des } \\
\text { gardons }\end{array}$ & AbGar & $\pi$ & $\begin{array}{l}\text { OBERDORFF et HUGHES, } 1992 ; \text { OBERDORFF et } \\
\text { PORCHER, } 1994 ; \text { PENCZAK et KOSZALINSKA, } 1993 \text {; } \\
\text { SCHIEMER et WAIDBACHER, } 1992\end{array}$ \\
\hline
\end{tabular}


bassin versant (transformée en $\log _{10}(x)$ ) et la pente (transformée en In $(x+1)$ ). Selon le résultat obtenu, deux cas sont alors envisagés:

- aucun des deux descripteurs du gradient n'entre dans la régression pas à pas. La comparaison entre les deux groupes de station se fait alors directement sur les valeurs prises par le paramètre au moyen d'un test-t de STUDENT (seuil : $p>0,05$ ),

- au moins un des descripteurs entre dans la régression pas à pas. Dans ce cas, la comparaison entre les deux groupes de station est effectuée sur les résidus de la régression. Un test-t de STUDENT sur les résidus obtenus par chacun des deux groupes de station est alors utilisé.

\section{RÉSULTATS ET DISCUSSION}

\section{Évolution amont-aval des paramètres}

Seuls le nombre d'espèces allochtones et le nombre d'espèces limnophiles sont indépendants du gradient amont/aval (Tableau III). Les 21 autres paramètres montrent au contraire une évolution amont/aval en liaison, soit avec la surface du bassin versant seule (Nesp, NeN, NeFp, NeFpNr, NeRh, NeEu, Ablit, AbPhy, AbPhyNr, AbR, AbReu et AbGar), soit avec la pente seule (AblnNr, NePeNr), soit avec les deux conjointement (Abln, AbPi, AbOm, NePE, AbLitNr, AbPhyLit, NeS) (Tableau III).

L'indépendance du nombre d'espèces allochtones s'explique par la distribution particulière de ces espèces davantage liées à des facteurs humains (introductions et/ou repeuplements) qu'à des facteurs environnementaux. Celle du nombre d'espèces limnophiles est sans doute due à leur faible représentation sur notre domaine d'étude réduit aux parties amont et médianes des cours d'eau (bassin versant $\leq 4140 \mathrm{~km}^{2}$ ) et à la discordance vis-à-vis du gradient amontaval des épinoches (Gasterosteus aculeatus) et épinochettes (Pungitius pungitius) déjà mis en évidence sur la Seine (BELLIARD, 1994).

Comme pour de nombreux bassins européens, la taille et la pente des rivières de notre domaine d'étude évoluent de manière conjointe et inverse le long du gradient amontaval $\left(\mathrm{r}^{2}=0,42\right)$. II nous est donc impossible de comprendre séparément l'effet de chacun des facteurs.

Cependant, globalement, les évolutions fonctionnelles des peuplements sont conformes à celles attendues. L'augmentation du nombre d'espèces de l'amont vers l'aval s'accompagne d'une diversification des stratégies de vie. Les peuplements sont dominés à l'amont par des invertivores, des espèces de fond pierreux, des rhéophiles et des lithophiles. Ils évoluent le long du gradient en présentant des espèces aux régimes trophiques, aux habitats d'alimentation, aux cycles de vie et aux substrats de ponte plus variés : l'abondance relative des omnivores, des piscivores, le nombre d'espèces de pleine eau, celui des eurytopes, l'abondance relative des phytophiles et des phyto-lithophiles augmentent vers l'aval au détriment de l'abondance relative des invertivores et des lithophiles (Tableau III). Cette diversification s'explique notamment par la complexification du réseau de transfert de l'énergie (VANNOTE et al., 1980 ; MINSHALL et al., 1985) et l'augmentation de la diversité de l'habitat (GORMAN et KARR, 1978 ; SCHLOSSER, 1982b) accompagnées de l'accroissement de la stabilité ou de la prévisibilité temporelle des variations du milieu, en particulier de l'hydrologie (HORWITZ, 1978 ; ZALEWSKI et NAIMAN, 1985).

Parallèlement à ces évolutions fonctionnelles, le nombre d'espèces sensibles et l'abondance relative de la composante résistante des peuplements (AbR, AbReu et AbGar) augmentent également vers l'aval avec la richesse globale du peuplement. 


\section{Tableau III}

Résultats des régressions multiples pas à pas de chacun des 23 paramètres sur les deux descripteurs de position des stations : la surface du bassin versant (transformée en $\log _{10}(X)$ ) et la pente (transformée en In $(X+1)$ ). Descripteur entrant $(p<0,05)$, pente, coefficient standard et probabilité des corrélations partielles.

\section{Table III}

Results of stepwise multiple regressions between each of the 23 parameters and the two position variables : catchment area $\left(\log _{10}(X)\right.$ transformed) and gradient $($ In $(X+1)$ transformed). Retained variable $(p<0.05)$, slope and standard coefficient and probability of partial correlations.

\begin{tabular}{|c|c|c|c|c|c|}
\hline $\begin{array}{l}\text { CARACTERISTIQUE } \\
\text { Paramètre } \\
\end{array}$ & Code & $\begin{array}{l}\text { Descripteur } \\
\text { entrant }\end{array}$ & Pente & $\begin{array}{r}\text { Coefficient } \\
\text { standard }\end{array}$ & Probabilité \\
\hline $\begin{array}{l}\text { RICHESSE GLOBALE } \\
\text { Nombre d'espèces }\end{array}$ & Nesp & BV & 2,812 & 0,396 & 0,0001 \\
\hline $\begin{array}{l}\text { Nombre d'espèces } \\
\text { autochtones }\end{array}$ & $\mathrm{NeN}$ & BV & 2,860 & 0.409 & 0,0001 \\
\hline Nombre d'espèces allochtones & $\mathrm{NeA}$ & Aucun & & & \\
\hline $\begin{array}{l}\text { RÉGIME TROPHIQUE } \\
\text { Abondance relative des } \\
\text { invertivores }\end{array}$ & Abln & $\begin{array}{l}\text { Pente } \\
\text { BV }\end{array}$ & $\begin{array}{r}0,215 \\
-0,163\end{array}$ & $\begin{array}{r}0,266 \\
-0,245\end{array}$ & $\begin{array}{l}0,0010 \\
0,0030\end{array}$ \\
\hline $\begin{array}{l}\text { Abondance relative des } \\
\text { invertivores non résistants }\end{array}$ & AblnNr & Pente & 0,313 & 0,395 & 0,0001 \\
\hline $\begin{array}{l}\text { Abondance relative des } \\
\text { piscivores }\end{array}$ & $\mathrm{AbPi}$ & $\begin{array}{l}\text { Pente } \\
\text { BV }\end{array}$ & $\begin{array}{r}-0,064 \\
0,040\end{array}$ & $\begin{array}{r}-0,249 \\
0,192\end{array}$ & $\begin{array}{l}0,0030 \\
0,0230\end{array}$ \\
\hline $\begin{array}{l}\text { Abondance relative des } \\
\text { omnivores }\end{array}$ & AbOm & $\begin{array}{l}\text { BV } \\
\text { Pente }\end{array}$ & $\begin{array}{r}0,127 \\
-0,148\end{array}$ & $\begin{array}{r}0,210 \\
-0,200\end{array}$ & $\begin{array}{l}0,0148 \\
0,0200\end{array}$ \\
\hline $\begin{array}{l}\text { HABITAT D'ALIMENTATION } \\
\text { Nombre d'especes de fond } \\
\text { pierreux }\end{array}$ & NeFp & BV & 0,670 & 0,311 & 0,0001 \\
\hline $\begin{array}{l}\text { Nombre d'espèces non } \\
\text { résistantes de fond pierreux }\end{array}$ & NeFpNr & BV & 0,596 & 0,359 & 0,0001 \\
\hline $\begin{array}{l}\text { Nombre d'espèce de pleine } \\
\text { eau }\end{array}$ & $\mathrm{NePe}$ & $\begin{array}{l}\text { BV } \\
\text { Pente }\end{array}$ & $\begin{array}{r}1,290 \\
-1,079\end{array}$ & $\begin{array}{r}0,312 \\
-0,215\end{array}$ & $\begin{array}{l}0,0001 \\
0,0083\end{array}$ \\
\hline $\begin{array}{l}\text { Nombre d'espèces non } \\
\text { résistantes de pleine eau }\end{array}$ & $\mathrm{NePeNr}$ & Pente & $-1,141$ & $-0,345$ & 0,0001 \\
\hline $\begin{array}{l}\text { CYCLE DE VIE } \\
\text { Nombre d'espèces rhéophiles }\end{array}$ & $\mathrm{NeRh}$ & BV & 1,261 & 0,303 & 0,0001 \\
\hline Nombre d'espèces limnophiles & Neli & Aucun & & & \\
\hline Nombre d'espèces eurytopes & $\mathrm{NeEu}$ & BV & 1,738 & 0,490 & 0,0001 \\
\hline $\begin{array}{l}\text { SUBSTRAT DE PONTE } \\
\text { Abondance relative des } \\
\text { lithophiles }\end{array}$ & AbLit & BV & $-0,214$ & $-0,364$ & 0,0001 \\
\hline $\begin{array}{l}\text { Abondance relative des } \\
\text { lithophiles non résistants }\end{array}$ & AbLitNr & $\begin{array}{l}\text { Pente } \\
\text { BV }\end{array}$ & $\begin{array}{r}0,149 \\
-0,118\end{array}$ & $\begin{array}{r}0,196 \\
-0,189\end{array}$ & $\begin{array}{l}0,0235 \\
0,0291\end{array}$ \\
\hline $\begin{array}{l}\text { Abondance relative des } \\
\text { phytophiles }\end{array}$ & AbPhy & BV & 0,034 & 0,300 & 0,0001 \\
\hline $\begin{array}{l}\text { Abondance relative des } \\
\text { phytophiles non résistants }\end{array}$ & AbPhyNr & $B V$ & 0,032 & 0,291 & 0,0001 \\
\hline $\begin{array}{l}\text { Abondance relative des phyto- } \\
\text { lithophiles }\end{array}$ & AbPhylit & $\begin{array}{l}\text { BV } \\
\text { Pente }\end{array}$ & $\begin{array}{r}0.149 \\
-0,120\end{array}$ & $\begin{array}{r}0,318 \\
-0,211\end{array}$ & $\begin{array}{l}0,0001 \\
0.0096\end{array}$ \\
\hline RÉSISTANCE/SENSIBILITÉ & & & & & \\
\hline Nombre d'espèces sensibles & $\mathrm{NeS}$ & $\begin{array}{l}\text { BV } \\
\text { Pente }\end{array}$ & $\begin{array}{r}0,389 \\
-0,438\end{array}$ & $\begin{array}{r}0,190 \\
-0,176\end{array}$ & $\begin{array}{l}0,0293 \\
0,0434\end{array}$ \\
\hline $\begin{array}{l}\text { Abondance relative des } \\
\text { individus résistants }\end{array}$ & $A b R$ & $B V$ & 0,144 & 0,261 & 0,0001 \\
\hline $\begin{array}{l}\text { Abondance relative des } \\
\text { individus résistants euryèces }\end{array}$ & AbReu & $B V$ & 0,129 & 0,349 & 0,0001 \\
\hline $\begin{array}{l}\text { Abondance relative des } \\
\text { gardons }\end{array}$ & AbGar & BV & 0,117 & 0,328 & 0,0001 \\
\hline
\end{tabular}




\section{Les paramètres sensibles aux dégradations}

Indépendamment du gradient amont/aval, 8 paramètres montrent des valeurs différentes entre les stations faiblement et fortement anthropisées (t-test, $p<0,05$ ) (Tableau IV). Ces 8 paramètres (AbInNr, NeFp, NeFpNr, NePeNr, NeRh, AbLit, AbLitNr et NeS) sont basés sur des espèces spécialisées ou sensibles et leurs valeurs décroissent avec l'augmentation du degré d'anthropisation conformément aux hypothèses préalables (Tableau IV).

Le nombre d'espèces sensibles (NeS) étant un indicateur global du degré de dégradation, il est impossible de relier son évolution à une cause directe. En revanche, chacun des 7 autres paramètres est révélateur d'un certain type de dégradation.

L'évolution de l'abondance relative des invertivores non résistants ( $\mathrm{Ab} / \mathrm{nNr}$ ) (Tableau IV) est conforme aux résultats obtenus sur d'autres milieux (KARR et al., 1986 ; GATZ et HARIG, 1993 ; OBERDORFF et PORCHER, 1994). Ces espèces sont sensibles à la diminution du stock d'invertébrés. Cette diminution peut être due à l'altération de la qualité de l'eau, des sources d'énergie (KARR et SCHLOSSER, 1978) ou des habitats disponibles (KARR et al., 1986 ; HARRIS, 1995).

Trois paramètres concernant l'habitat d'alimentation, le nombre d'espèces de fond pierreux (NbFp), le nombre d'espèces non résistantes de fond pierreux ( $\mathrm{NbFpNr}$ ), et le nombre d'espèces non résistantes de pleine eau ( $\mathrm{NePeNr}$ ), montrent une sensibilité significative vis-à-vis du degré d'anthropisation (Tableau IV). Les espèces de fond pierreux sont fortement affectées par le colmatage des milieux de type radier qui induit une baisse de la disponibilité de nourriture, en particulier du stock d'insectes (BERKMAN et RABENI, 1987 ; RABENI et SMALE, 1995). Les espèces de pleine eau sont en revanche exigeantes concernant les vitesses de courant (GRANDMOTTET, 1983 ; KARR et al., 1986) et sont donc sensibles à la perturbation des écoulements que provoquent les barrages, les travaux de recalibrage ou de canalisation.

La baisse du nombre d'espèces rhéophiles dans les milieux fortement anthropisés (Tableau IV) confirme les résultats obtenus sur d'autres cours d'eau européens (SCHIEMER et WAIDBACHER, 1992 ; PENAZ et JURAJDA, 1993). Le développement de ces espèces qui effectuent la totalité de leur cycle de vie exclusivement dans le chenal requiert en effet une qualité et une diversité élevées des habitats de berge. Ces espèces exigent en particulier pendant les phases larvaire et juvénile, une succession de baies protégées du courant et de plages de gravier peu profondes (SCHIEMER et al., 1991). Elles sont ainsi très sensibles à l'homogénéisation des berges qu'entraînent les travaux de recalibrage et de régulation. Sur le Danube, par exemple, SCHIEMER et al. (1991) estiment qu'environ $60 \%$ du linéaire de la partie autrichienne est totalement défavorable aux jeunes d'espèces rhéophiles suite aux aménagements.

La sensibilité des espèces lithophiles (AbLit et de AbLitNr) (Tableau IV) provient de la forte vulnérabilité au colmatage des plages de sable ou graviers sur lesquelles ces espèces déposent leur ponte. Ce colmatage induit en effet une baisse de l'oxygénation de la couche d'eau profonde pouvant entraîner une forte mortalité des pontes (KARR et al., 1986 ; BERKMAN et RABENI, 1987). Des résultats comparables ont été obtenus sur une large variété de cours d'eau (BERKMAN et RABENI, 1987 ; PENCZAK et MANN, 1993 ; OBERDORFF et PORCHER, 1994 ; RABENI et SMALE, 1995).

Toutefois, il est important de noter qu'en raison de la faible richesse spécifique du bassin de la Seine, ces 8 paramètres sensibles aux dégradations possèdent de nombreuses espèces communes. Ainsi 4 parmi les 6 espèces invertivores non résistantes, 5 parmi les 6 espèces de fond pierreux (4 sur les 4 espèces non résistantes de fond 


\section{Tableau IV}

Sensibilité des 23 paramètres au degré d'anthropisation. Résultat des test-t de STUDENT sur les valeurs des paramètres (pour les paramètres avec •) ou des résidus de la régression multiple pas à pas (pour les 21 autres paramètres) entre les stations faiblement et fortement anthropisées. Sens de variation avec l'augmentation du degré d'anthropisation, valeur du test-t et probabilité. Ns : test non significatif $(p>0,05)$.

\section{Table IV}

Sensitivity to the rivers alteration of the 23 parameters. Results of the STUDENT's t-test on the parameters values (for parameters with $\%$ ) or on the residues of stepwise regression (for the 21 other parameters) between the slightly and strongly disturbed sites. Variation according to the degree of perturbation, STUDENT's test value and probability. Ns : no significant test $(p>0.05)$.

\begin{tabular}{|c|c|c|c|c|}
\hline $\begin{array}{l}\text { CARACTÉRISTIQUE } \\
\text { Paramêtre }\end{array}$ & Code & $\begin{array}{c}\text { Variation } \\
\text { avec le } \\
\text { degré de } \\
\text { dégradation }\end{array}$ & $\begin{array}{l}\text { Valeur } \\
\text { test-t }\end{array}$ & Probabilité \\
\hline $\begin{array}{l}\text { RICHESSE GLOBALE } \\
\text { Nombre d'espèces }\end{array}$ & Nesp & NS & 1,246 & 0,2140 \\
\hline $\begin{array}{l}\text { Nombre d'espèces } \\
\text { autochtones }\end{array}$ & $\mathrm{NeN}$ & NS & 0,497 & 0,6197 \\
\hline * Nombre d'espèces allochtones & $\mathrm{NeA}$ & NS & 0,544 & 0,5869 \\
\hline $\begin{array}{l}\text { RÉGIME TROPHIQUE } \\
\text { Abondance relative des } \\
\text { invertivores. }\end{array}$ & Abln & NS & 1,264 & 0,2078 \\
\hline $\begin{array}{l}\text { Abondance relative des } \\
\text { invertivores non résistants }\end{array}$ & AblnNr & $x$ & 2,866 & 0,0046 \\
\hline $\begin{array}{l}\text { Abondance relative des } \\
\text { piscivores }\end{array}$ & $\mathrm{AbPi}$ & NS & $-0,647$ & 0,5184 \\
\hline $\begin{array}{l}\text { Abondance relative des } \\
\text { omnivores }\end{array}$ & AbOm & NS & $-1,196$ & 0,2331 \\
\hline $\begin{array}{l}\text { HABITAT D'ALIMENTATION } \\
\text { Nombre d'especes de fond } \\
\text { pierreux }\end{array}$ & $\mathrm{NeFp}$ & $x$ & 1,941 & 0,0536 \\
\hline $\begin{array}{l}\text { Nombre d'espèces non } \\
\text { résistantes de fond pierreux }\end{array}$ & $\mathrm{NeFpNr}$ & $x$ & 3,005 & 0,0030 \\
\hline $\begin{array}{l}\text { Nombre d'espèce de pleine } \\
\text { eau }\end{array}$ & $\mathrm{NePe}$ & NS & 1,639 & 0,1028 \\
\hline $\begin{array}{l}\text { Nombre d'espèces non } \\
\text { résistantes de pleine eau }\end{array}$ & NePeNr & $x$ & 2,031 & 0,0435 \\
\hline $\begin{array}{l}\text { CYCLE DE VIE } \\
\text { Nombre d'espèces rhéophiles }\end{array}$ & $\mathrm{NeRh}$ & 2 & 2,618 & 0,0095 \\
\hline * Nombre d'espèces limnophiles & $\mathrm{NeLi}$ & NS & $-0,632$ & 0,5284 \\
\hline Nombre d'espèces eurytopes & $\mathrm{NeEu}$ & NS & $-0,223$ & 0,8234 \\
\hline $\begin{array}{l}\text { SUBSTRAT DE PONTE } \\
\text { Abondance relative des } \\
\text { lithophiles }\end{array}$ & AbLit & $\mathbf{x}$ & 2,237 & 0,0264 \\
\hline $\begin{array}{l}\text { Abondance relative des } \\
\text { lithophiles non résistants }\end{array}$ & AbLitNr & $x$ & 2,900 & 0,0041 \\
\hline $\begin{array}{l}\text { Abondance relative des } \\
\text { phytophiles }\end{array}$ & AbPhy & NS & $-0,385$ & 0,7009 \\
\hline $\begin{array}{l}\text { Abondance relative des } \\
\text { phytophiles non résistants }\end{array}$ & AbPhyNr & NS & $-0,406$ &, 6855 \\
\hline $\begin{array}{l}\text { Abondance relative des phyto- } \\
\text { lithophiles }\end{array}$ & AbPhylit & NS & $-0,829$ & .4083 \\
\hline $\begin{array}{l}\text { RÉSISTANCE/SENSIBILITÉ } \\
\text { Nombre d'especes sensibles }\end{array}$ & $\mathrm{NeS}$ & 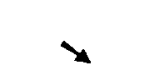 & 4,225 & 0,0001 \\
\hline $\begin{array}{l}\text { Abondance relative des } \\
\text { individus resistants }\end{array}$ & $A b R$ & NS & $-1,642$ & 0,1008 \\
\hline $\begin{array}{l}\text { Abondance relative des } \\
\text { individus résistants euryèces }\end{array}$ & AbReu & NS & $-0,078$ & 0,9381 \\
\hline $\begin{array}{l}\text { Abondance relative des } \\
\text { gardons }\end{array}$ & AbGar & NS & $-0,029$ & 0,9772 \\
\hline
\end{tabular}


pierreux), 12 parmi les 15 espèces rhéophiles et 7 parmi les 12 espèces non résistantes de pleine eau sont également des espèces lithophiles (Tableau I). Or, du fait de ce chevauchement, la sensibilité d'un paramètre peut provenir de l'altération d'un compartiment autre que celui visé. On peut en effet supposer que les poissons sont plus affectés par la dégradation de leur habitat de ponte que par la baisse de la disponibilité de leur alimentation (BALON, 1975b ; BALON, 1975a), ou des habitats favorables à leur alimentation. SCHIEMER et WAIDBACHER (1992) notent ainsi que sur le Danube les caractéristiques écologiques les plus signifiantes des espèces en danger sont les exigences des stades larvaires et juvéniles.

\section{Les paramètres insensibles aux dégradations}

Quinze paramètres ne montrent aucune différence entre les stations faiblement et fortement anthropisées (t-test, $p>0,05$ ) (Tableau IV). Ils n'apparaissent donc pas sensibles au degré d'anthropisation du milieu contrairement aux hypothèses et aux résultats observés sur d'autres systèmes.

Cette insensibilité peut avoir trois causes différentes : la particularité de la faune piscicole européenne, l'ampleur et la variété des altérations subies par les rivières du bassin, et la construction des paramètres eux-mêmes.

- Malgré leur origine commune, les faunes piscicoles d'Amérique du Nord et d'Europe présentent des stratégies biologiques contrastées (MAHON, 1984). En Europe, la faune piscicole est marquée par l'abondance d'espèces généralistes, souvent de grande taille, capables d'effectuer d'importants déplacements à l'intérieur du réseau fluvial. En outre, la fécondité des espèces est souvent importante. A l'inverse, en Amérique du Nord, les petites espèces sédentaires et fortement spécialisées sont nombreuses. La faible fécondité de la plupart d'entre elles est compensée par des stratégies de protection de la ponte. Dans un tel contexte, il est donc logique que les peuplements piscicoles nord-américains et européens montrent des réactions différentes vis-à-vis de l'altération des milieux. Par ailleurs, la relativement faible spécialisation des espèces européennes implique une sensibilité moindre des peuplements face à des modifications de leur environnement.

- Par ailleurs, les différents paramètres décrivant les peuplements répondent à des stades d'altération qui leur sont spécifiques (KARR et al., 1986 ; LEONARD et ORTH, 1986). Alors que certains d'entre eux ne sont sensibles qu'aux premiers stades d'altération, d'autres au contraire ne varient que pour des degrés d'altération plus avancés. Sur le bassin de la Seine, l'ensemble des cours d'eau a déjà été touché par des activités humaines. De ce fait, il est donc probable que la gamme de sensibilité de certains paramètres soit déjà dépassée.

En outre, la réponse des peuplements piscicoles vis-à-vis des interventions humaines dépend avant tout des conséquences de ces interventions sur les écosystèmes. Ainsi, STEEDMAN (1988) note que les densités ne varient pas de manière monotone avec l'intensité des dégradations et que les milieux moyennement et fortement dégradés présentent des densités respectivement supérieures et inférieures à celles des milieux naturels. De même, BELLIARD et al. (1995) sur la Seine mettent en évidence au cours du siècle dernier, des évolutions de peuplements inverses selon les types d'aménagement. En l'absence de connaissance précise sur le type de dégradation subi par les stations étudiées, on ne peut donc pas écarter l'existence de tels mécanismes pour expliquer l'absence de sensibilité de certains paramètres.

- Enfin, à cause de la faible richesse spécifique du peuplement (OBERDORFF et al., 1997), certains paramètres semblent constitués d'un nombre insuffisant d'espèces pour que leur sensibilité ne soit masquée par leur variabilité naturelle. C'est sans doute le cas par exemple de $\mathrm{NeA}, \mathrm{AbPi}$, Neli, AbPhy. 
De plus, la sensibilité de certains paramètres peut être également réduite par une trop forte hétérogénéité biologique des espèces (ANGERMEIER et KARR, 1986 ; HUGHES et GAMMON, 1987 ; STEEDMAN, 1988). Sur le bassin de la Seine par exemple, l'abondance relative des piscivores (AbPi) ne répond pas au degré d'anthropisation des stations. Nous pensons que cette situation s'explique par l'hétérogénéité et les particularités biologiques des espèces qui constituent ce groupe (Tableau I) : la lote (Lota lota) est sensible, le black-bass (Micropterus salmoïdes) est sensible et allochtone, le sandre (Stizostedion lucioperca) est résistant et le brochet (Esox lucius) est une espèce fortement manipulée. Cette hypothèse est confirmée par le résultat des paramètres nombre d'espèces de pleine eau ( $\mathrm{NePe}$ ) et abondance relative des invertivores (Abln) qui ne deviennent sensibles qu'après élimination des espèces résistantes (paramètres NePeNr et AblnNr) (Tableau IV).

\section{CONCLUSION}

Nos résultats mettent en évidence la sensibilité des peuplements piscicoles français vis-à-vis des dégradations. D'ores et déjà, ces résultats constituent une base pour évaluer la qualité d'un peuplement piscicole et prévoir en partie les impacts anthropiques. A terme, ils pourront participer au fondement d'un indice de qualité des milieux. Dans une telle optique, il conviendrait de préciser les modifications des peuplements piscicoles induites par les différents types d'altération des cours d'eau.

Cependant, en raison de ses caractéristiques, en particulier la pauvreté et la faible spéciation de son peuplement, le bassin de la Seine ne semble pour l'instant pas favorable au transfert direct de résultats établis sur d'autres bassins dans le cadre de l'IBI. Seuls huit paramètres apparaissent sensibles au degré de dégradation des cours d'eau : l'abondance relative des invertivores non résistants, le nombre d'espèces (non résistantes) de fond pierreux, le nombre d'espèces non résistantes de pleine eau, le nombre d'espèces rhéophiles, l'abondance relative des lithophiles (non résistants) et le nombre d'espèces sensibles. En outre, la plupart de ces paramètres sont liés entre eux par un nombre important d'espèces communes. Ces faits constituent sans doute une limite importante à la constitution d'indices fondés sur les peuplements piscicoles.

La difficulté du choix de paramètres sensibles à la dégradation avait déjà été mise en évidence dans le cas d'un peuplement pauvre en espèces (26 espèces) par BRAMBLETT et FAUSCH (1991) sur une rivière des Grandes Plaines de l'ouest des États-Unis. Les auteurs conseillaient alors comme approche alternative de prendre en compte des taxons complémentaires comme les macroinvertébrés benthiques.

Nous pensons que sur la Seine la pauvreté et la faible spéciation du peuplement peuvent être compensées en premier lieu par la prise en compte pour chacune des espèces des différents stades de développement. II semble que pour ce faire, ceux associés au développement des jeunes poissons soient des plus favorables (SCHIEMER et SPINDLER, 1989). C'est en effet durant leurs premiers stades de développement que les poissons, du fait de leur moindre mobilité, sont les plus dépendants de leur habitat (COPP et al., 1991; SCHLOSSER, 1991). C'est aussi au cours de cette période que les poissons ont les exigences les plus étroites (SCHIEMER et al., 1991).

\section{REMERCIEMENTS}

Les auteurs remercient E. TALES et les relecteurs anonymes pour la pertinence de leurs remarques. Les pêches ont été effectuées par le CSP et le Cemagref. 


\section{BIBLIOGRAPHIE}

ANGERMEIER P.L., KARR J.R., 1986. Applying an Index of Biotic Integrity based on stream-fish communities : considerations in sampling and interpretation. North Am. J. Fisheries Manag., 6, 418-429.

AREA, 1992. Cartographie de synthèse des schémas départementaux de vocation piscicole et des Z.N.I.E.F.F. humides du bassin Seine-Normandie. Commission de Bassin Seine-Normandie, $64 \mathrm{p}$.

BALON E.K., 1975a. Ecological guilds of fishes : a short summary of the concept and its application. Verh. Int. Ver. Limnol., 19, 2430-2439.

BALON E.K., 1975b. Reproductive guilds of fishes : a proposal and definition. J. Fish. Res. Board Can., 32, 821-864.

BALON E.K., 1981. Additions and amendments to the classification of reproductive styles in fishes. Environ. Biol. Fish., 6, 377-389.

BANARESCU P.M., 1989. Zoogeography and history of the freshwater fish fauna of Europe. In HOLCIK J. (ed.), The freshwater fishes of Europe, 88-107, Aula-verlag, Wiesbaden.

BELLIARD J., 1994. Le peuplement ichtyologique du bassin de la Seine : rôle et signification des échelles temporelle et spatiale. Thèse de doctorat, Paris VI, $197 \mathrm{p}$.

BELLIARD J., BOËT P., ALLARDI J., 1995. Évolution à long terme du peuplement piscicole du bassin de la Seine. Bull. Fr. Pêche et Pisciculture, 337/338/339, 83-91.

BELLIARD J., BOËT P., TALES E., 1997. Regional and longitudinal patterns of fish community structure of the Seine basin, France. Environ. Biol. Fish., 50, 133-147.

BERKMAN H.E., RABENI C.F., 1987. Effect of siltation on fish communities. Environ. Biol. Fish., 18, 285-294.

BERREBI dit THOMAS R., 1994. Étude critique d'un indice biotique d'intégrité des cours d'eau fondé sur les peuplements de poissons. Proposition d'un nouvel indice pour la Seine. Rapport DEA, Paris VI, $50 \mathrm{p}$.

BRAMBLETT R.G., FAUSCH K.D., 1991. Variable fish communities and the Index of Biotic Integrity in a western Great Plains river. Tree., 120, 752-769.

CHANGEUX T., 1995. Structure du peuplement piscicole à l'échelle d'un grand bassin européen : organisation longitudinale, influence de la pente et tendances régionales. Bull. Fr. Pêche et Pisciculture, 337/338/339, 63-74.

COPP G.H., 1991. Typology of aquatic habitats in the great Ouse, a small regulated lowland river. Regulated Rivers, 6, 125-134.

COPP G.H., OLIVIER J.M., PENAZ M., ROUX A.L., 1991. Juvenile fishes as functional describers of fluvial ecosystem dynamics : applications on the river Rhône, France. Regulated Rivers, 6, 135-145.

CRUMBY W.D., WEBB M.A., BULOW F.J., 1990. Changes in biotic integrity of a river in north-central Tennessee. Trans. Am. Fish. Soc., 119, 885-893.

DEGIORGI F., RAYMOND J.C., 1995. Réseau National de Bassin. Mise en place du suivi piscicole : 1994. Conseil Supérieur de la Pêche, Agence de l'eau RhôneMéditerranée-Corse, $60 \mathrm{p}$.

DUPIAS G., REY P., 1985. Document pour un zonage des régions phyto-écologiques. CNRS, Toulouse, $39 \mathrm{p}$.

FAUSCH K.D., LYONS J., KARR J.R., ANGERMEIER P.L., 1990. Fish communities as indicators of environmental degradation. American Fisheries Society Symposium, 123-144.

GATZ A.J., HARIG A.L., 1993. Decline in the index of biotic integrity of Delaware Run, over 50 years. Ohio journal of science, 4, 95-100. 
GORMAN O.T., KARR J.R., 1978. Habitat structure and stream fish communities. Ecology, 59, 507-515.

GRANDMOTTET J.P., 1983. Principales exigences des téléostéens dulcicoles vis-à-vis de I'habitat aquatique. Annales Scientifiques de l'Université de Franche-Comté, Besançon, 4, 3-32.

HARRIS J.H., 1995. The use of fish in ecological assessments. Australian Journal of Ecology, $20,65-80$.

HOLCIK J., 1990. Effects of hydraulic engineering on habitat and fish community in river anabranches of the middle Danube. In VAN DENSEN W.L.T., STEINMETZ B., HUGHES R.H. (eds), Management of freshwater fisheries, Proceedings of a symposium organized by the European inland fisheries advisory commission, 14-24, Pudoc Wageningen, Göteborg, Sweden.

HORWITZ R.J., 1978. Temporal variability patterns and the distributional patterns of stream fishes. Ecol. Monogr., 48, 307-321.

HUET M., 1949. Aperçu des relations entre la pente et les populations piscicoles des eaux courantes. Revue Suisse d'Écologie, XI, 332-351.

HUGHES R.M., GAMMON J.R., 1987. Longitudinal changes in fish assemblages and water quality in the Willamette river, Oregon. Trans. Am. Fish. Soc., 116, 196-209.

HUGHES R.M., OBERDORFF T., 1997. Applications of IBI concepts and metrics to waters outside the United States and Canada. In SIMON T.P. (ed.), Assessment approaches for estimating biological integrity using fish assemblages, Lewis Press, Bocca Raton, FL, USA, sous presse.

JURAJDA P., 1995. Effect of channelization and regulation on fish recruitment in a flood plain river. Regul. Rivers, 10, 207-215.

KARR J.R., 1981. Assessment of biotic integrity using fish communities. Fisheries, 6, 21-27.

KARR J.R., 1993. Defining and assessing ecological integrity : beyond water quality. Environmental toxicology and chemistry, 12, 1521-1531.

KARR J.R., SCHLOSSER I.J., 1978. Water resources and the land-water interface. Science, 201, 229-234.

KARR J.R., FAUSCH K.D., ANGERMEIER P.L., YANT P.R., SCHLOSSER I.J., 1986. Assessing biological integrity in running waters. A method and its rationale. Illinois natural history survey, Special publication, 5, 1-28.

LAMONTAGNE M., BARIBEAU T., 1992. L'évaluation comparative des grands fleuves. Revue de géographie de Lyon, 68 4/92, 265-272.

LEIDY R.A., FIELDER P.L., 1985. Human disturbance and patterns of fish species diversity in the San Francisco bay drainage, California. Environmental conservation, 33, 247-267.

LEONARD P.M., ORTH D.J., 1986. Application and testing of an index of biotic integrity in small, coolwater streams. Trans. Am. Fish. Soc., 115, 401-414.

MAHON R., 1984. Divergent structure in fish taxocenes of north temperate streams. Can. J. Fish. Aqu. Sci., 41, 330-350.

MICHEL P., OBERDORFF T., 1995. Feeding habits of fourteen European freshwater fish species. Cybium, 19, 5-46.

MILLER D.L., LEONARD P.M., HUGHES R.M., KARR J.R., MOYLE P.B., SCHRADER L.H., THOMPSON B.A., DANIELS R.A., FAUSCH K.D., FITZHUGH G.A., GAMMON J.R., HALLIWELL D.B., ANGERMEIER P.L., ORTH D.J., 1988. Regional applications of Index of Biotic Integrity for use in water resource management. Fisheries, 13, 12-20.

MINNS C.K., CAIRNS V.W., RANDALL R.G., MOORE J.E., 1994. An Index of Biotic Integrity (IBI) for fish assemblages in the littoral zone of Great Lakes areas of concern. Can. J. Fish. Aqu. Sci., 51, 1804-1822. 
MINSHALL G.W., CUMMINS K.W., PETERSEN R.C., CUSHING C.E., BRUNS D.A., SEDELL J.R., VANNOTE R.L., 1985. Developments in stream ecosystem theory. Can. J. Fish. Aqu. Sci., 42, 1045-1055.

OBERDORFF T., HUGHES R.M., 1992. Modification of an index of biotic integrity based on fish assemblages to characterize rivers of the Seine basin, France. Hydrobiologia, 228, 117-130.

OBERDORFF T., GUILBERT E., LUCCHETTA J.C., 1993. Patterns of fish species richness in the Seine River basin, France. Hydrobiologia, 259, 157-167.

OBERDORFF T., PORCHER J.P., 1994. An index of biotic integrity to assess biological impacts of salmonid farm effluents on receiving waters. Aquaculture, 119, 219-235.

OBERDORFF T., HUGUENY B., GUÉGUAN J.F., 1997. Is there an influence of the historical events on contemporary fish species richness in rivers ? Comparisons between Western Europe and North America. Journal of biogeography, 24, 113-148.

PENAZ M., JURAJDA P., 1993. Fish assemblages of the Morava River - Longitudinal zonation and protection. Folia Zool., 42, 317-328.

PENAZ M., OLIVIER J.M., CARREL G., PONT D., ROUX A.L., 1991. A synchronic study of juvenile fish assemblages in the French section of the Rhône River. Acta Scientiarum Naturalium Brno, 25, 1-36.

PENCZAK T., KOSZALINSKA M., 1993. Populations of dominant fish species in the Narew river under human impacts. Pol. Arch. Hydrobiol., 40, 59-75.

PENCZAK T., MANN R.H.K., 1993. A preliminary evaluation of the effect of human activity on the fish populations of the Pilica river, Central Poland. Pol. Arch. Hydrobiol., 40, 101-115.

RABENI C.F., SMALE M.A., 1995. Effect of siltation on stream fishes and potential mitigating role of the buffering riparian zone. Hydrobiologia, 303, 211-219.

SCHIEMER F., SPINDLER T., 1989. Endangered fish species of the Danube river in Austria. Regul. Rivers, 4, 397-407.

SCHIEMER F., WAIDBACHER H., 1992. Strategies for conservation of a Danubian fish fauna. In BOON P.J., CALOW P., PETTS G.E. (eds), River conservation and management, 363-382, John Wiley and sons.

SCHIEMER F., WIESER W., 1992. Epilogue : food and feeding, ecomorphology, energy assimilation and conversion in cyprinids. Environ. Biol. Fish., 33, 223-227.

SCHIEMER F.T., SPINDLER T., WINTERSBERGER H., SCHEINDER A., CHOVANEC A., 1991. Fish fry associations : important indicators for the ecological status of large rivers. Verh. Internat. Verein. Limnol., 24, 2497-2500.

SCHLOSSER I.J., 1982a. Trophic structure, reproductive success, and growth rate of fishes in a natural and modified headwater stream. Can. J. Fish. Aqu. Sci., 39, 968-978.

SCHLOSSER I.J., 1982b. Fish community structure and function along two habitat gradients in a headwater stream. Ecological Society of America, 52, 395-414.

SCHLOSSER I.J., 1991. Stream fish ecology : a landscape perspective. Bioscience, 41, 704-712.

SPILLMANN C.J., 1961. 65 poissons d'eau douce. Fédération française des sociétés des sciences naturelles, Paris, $303 \mathrm{p}$.

STEEDMAN R.J., 1988. Modification and assessment of an Index of Biotic Integrity to quantify stream quality in southern Ontario. Can. J. Fish. Aqu. Sci., 45, 492-501.

TROCHERIE F., SOUCHON Y., WASSON J.G., 1985. Note de réflexion concernant la mise en place d'objectifs relatifs à la gestion piscicole et halieutique des cours d'eau. Cemagref, Lyon, $9 \mathrm{p}$.

VANNOTE R.L., MINSHALL G.W., CUMMINS K.W., SEDELL J.R., CUSHING C.E., 1980. The river continuum concept. Can. J. Fish. Aqu. Sci., 37, 130-137. 
VERNEAUX J., 1981. Les poissons et la qualité des cours d'eau. Ann. Sci. Univ. FrancheComté, Besançon, Biol. Anim., 4, 33-41.

ZALEWSKI M., NAIMAN R.J., 1985. The regulation of riverine fish communities by a continuum of abiotic-biotic factors. In ALBASTER J.S. (ed.), Habitat modification and freshwater fisheries, 3-9, Butterworks Publication, London. 